\title{
Submitted: 7 Liver herniation into the pericardium mimicking Accepted: a pericardial tumor: unusual presentation of trisomy 13
} 06.09.2021

Published: 29.11.2021

\author{
Joanna Szymkiewicz-Dangel ${ }^{1}$, Maria Magdalena Hussey² \\ ${ }^{1}$ Department of Perinatal Cardiology and Congenital anomalies, Centre of Postgraduate \\ Medical Education, Warsaw, Poland \\ ${ }^{2}$ Emergency Department, Szpital Wolski im. Anny Gostynskiej, Warsaw, Poland \\ Correspondence: Joanna Szymkiewicz-Dangel, Department of Perinatal Cardiology and \\ Congenital anomalies, Centre of Postgraduate Medical Education, Agatowa 10, 03-680, \\ Warsaw,Poland; e-mail: joanna.dangel@gmail.com
}

DOI: $10.15557 / \mathrm{JoU} .2021 .0058$

Keywords

trisomy 13 , pericardial effusion, pericardial tumor,

diaphragmatic hernia, fetal echocardiography

\begin{abstract}
Aim of the study: Trisomy 13 is the third most common autosomal trisomy. The following case report shows an atypical case of trisomy 13, highlighting the usefulness of $3 \mathrm{D}$ volume storage and reconstruction, and the necessity of careful interpretation of the first trimester screening results. Case description: The results of the first trimester screening tests were interpreted as normal, and invasive tests were not recommended. At 21 weeks, a bright spot in the left ventricle was noted, and fetal echocardiography was performed at 33 weeks. The scan showed a massive pericardial effusion and a pericardial tumor located in front of the right ventricle. Conclusions: The final diagnosis, made postnatally, revealed an atypical right-sided diaphragmatic hernia. Part of the liver was displaced to the pericardial cavity, mimicking a pericardial tumor in a baby with trisomy 13. Following the diagnosis of the lethal disorder, the baby was discharged under a home-based palliative care program and died on the $49^{\text {th }}$ day of life.
\end{abstract}

\section{Case description}

A 34-year-old gravida 2 para 1 was referred for fetal echocardiography following a diagnosis of a hyperechogenic focus in the left ventricle at 22 weeks of gestation.

The patient was in her second single low-risk pregnancy. Her first daughter was healthy. A combined first trimester screening test was performed at 13 weeks. Fetal nuchal translucency (NT) equaled $1.8 \mathrm{~mm}$ for a crown-rump length (CRL) $72 \mathrm{~mm}$, with a fetal heart rate (FHR) of 164/ min, and no tricuspid valve regurgitation.

The fetus showed no signs of craniofacial abnormality, and the nasal bone was visible. A biochemical test presented decreased levels of beta-human chorionic gonadotrophin ( $\beta$-hCG) and pregnancy-associated plasma protein A (PAPP-A) down to $11.98 \mathrm{IU} / \mathrm{I}(0.395 \mathrm{MoM})$ and $0.867 \mathrm{IU} / \mathrm{I}$ (0.334 MoM), respectively. The background risk was 1:821 for trisomy 18 and 1:2565 for trisomy 13, and it there was a noticeable rise after the biochemical tests to 1:423 for trisomy 18 and 1:969 for trisomy 13. The risk of trisomy 21 significantly dropped from 1:324 to 1:3196. Despite the fact that the risk was recognized as moderate (higher than $1: 1000)$, the results were interpreted as normal. After a medical consultation by a doctor who held a certificate issued by the Fetal Medicine Foundation, the patient was scheduled for a second trimester ultrasound at around 20 weeks without any additional tests. No pathological findings were noticed except for a single bright spot in the left ventricle, so the woman was referred for fetal echocardiography. The fetus was growing consistently with gestational age.

Due to unknown reasons, the patient reported to the prenatal cardiology reference center at 33 weeks. The scan showed a massive pericardial effusion and a tumor located in front of the right ventricle. The anatomy and function of the heart were normal, with no signs of cardiac tamponade. The echogenicity of the suspicious mass resembled rhabdomyoma, lung or liver, and appeared atypical for a pericardial tumor (Fig. 1). Subsequently, a typical position of the abdominal organs and a continuity of the diaphragm were 


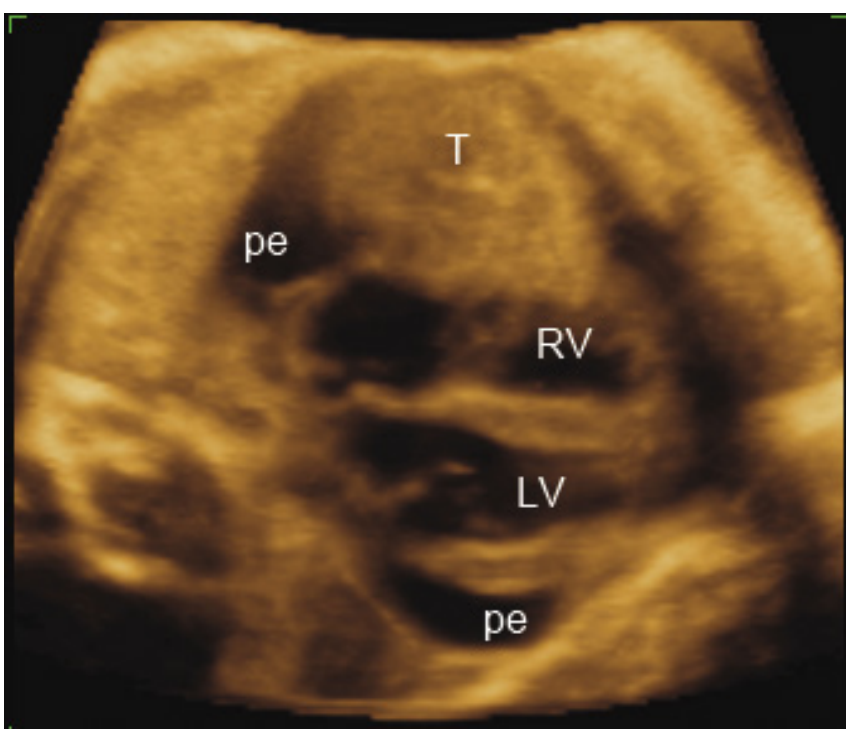

Fig. 1. Original two-dimensional echocardiography picture which was taken during the first echocardiography exam. Pericardial effusion (pe) is seen. Large pericardial tumor (T) is located in front to the right ventricle $(R V)$

revealed. Due to the unusual appearance of the pericardial tumor a magnetic resonance scan was planned in 3 weeks' time, but it was not performed due to the premature delivery. Ultrasound examination completed a week later (at 34 weeks) by an experienced perinatologist confirmed a mass measuring $30 \times 19 \mathrm{~mm}$ inside the pericardial cavity, and no need for fetal pericardiocentesis.

Spontaneous vaginal delivery occurred at 36 weeks at the perinatal referral center. The girl weighed 2,690 grams and scored 4 and 6 Apgar points at 1 and 5 minutes after birth, respectively. Due to respiratory distress she was intubated, without a need for catecholamines. Neonatal physical examination revealed dysmorphic features including benign cleft lip, which was beyond prenatal ultrasound diagnostic capabilities (Fig. 2), hypotelorism, flat nasal bridge, narrow, down-slanting palpebral fissures, and additional rudimentary toes in both feet and one finger in the right hand. The X-ray and echocardiography scan completed at the neonatal ICU appeared unclear and ambiguous. An atypical shadow was seen around the heart, however the diaphragm looked normal (Fig. 3). Consequently, the neonate was transported to the referral pediatric hospital at the first day of life.

She went through an additional ultrasound scan and MRI of the chest and abdomen. The results showed an atypical right diaphragmatic hernia with a part of the liver displaced to the pericardial cavity. Blood for the karyotype testing was collected the next day. On the $3^{\text {rd }}$ day of life, diaphragmatic hernia closing surgery and fluid evacuation from the pericardial cavity were performed, followed by the amputation of the rudimentary digits on day 6 after birth.

After the operation, the patient suffered from supraventricular tachycardia which was treated with Amiodarone. In the $3^{\text {rd }}$ week of life, the result of the karyotype testing

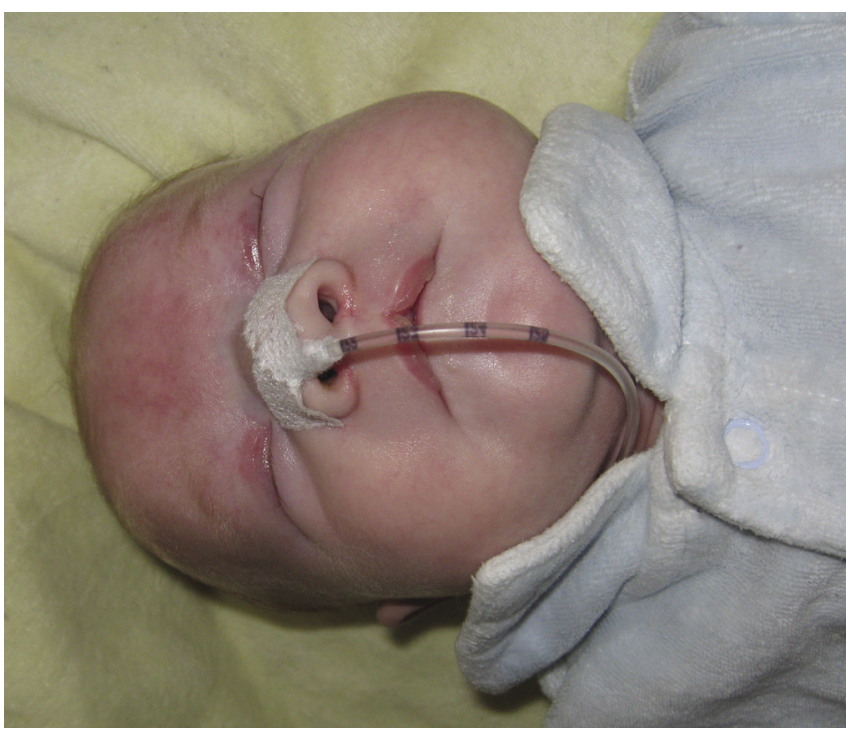

Fig. 2. Benign cleft lip in a baby with trisomy 13

confirmed the diagnosis of trisomy 13. Due to the lethal disorder, on day 26 after birth the patient was discharged from the hospital and admitted to the home care of the Warsaw Hospice for Children. She died asleep at home 4 weeks later.

When the final diagnosis was made, the fetal echocardiography scan was analyzed again using STIC method. A retrospective offline reconstruction visualized liver herniation into the pericardial cavity (Fig. 4). The picture showing a right diaphragmatic hernia most likely was unavailable during the standard prenatal ultrasound examination.

\section{Discussion}

Trisomy 13 is the third most common autosomal trisomy, with an estimated prevalence of 1:5000-1:30 000 live births ${ }^{(1,2)}$. Previous studies have indicated that the rate of occurrence rises up to 1:800 during the weeks 11-14 of pregnancy ${ }^{(3)}$. It has been reported that the effectiveness of screening for trisomy 13 at 10-14 weeks of gestation by a combination of maternal age, fetal NT and the maternal serum b-hCG and PAPP-A estimates around $84 \%-90 \% \%^{(4,5)}$. Invasive prenatal tests should be recommended when the specific risk of a chromosomal abnormality is higher than the risk of miscarriage. Up to 2017, according to the NHS recommendation for patients ${ }^{(6)}$, based on the guidelines prepared by the Royal College of Obstetricians and Gynecologists ${ }^{(7)}$, as well as the American ${ }^{(8)}$ and Canadian ${ }^{(9)}$ statements, the risk of amniocentesis was estimated at around $1 \%$. The current data, showing that amniocentesis does not increase risk of miscarriage over the background risk ${ }^{(10)}$, was not known at the time of counselling the patient.

So, according to the state of knowledge at the time of this report, the specific risk for trisomy 13 was around 10 times lower than that of the complications associated with invasive prenatal testing. Compared to the basic risk, it almost 


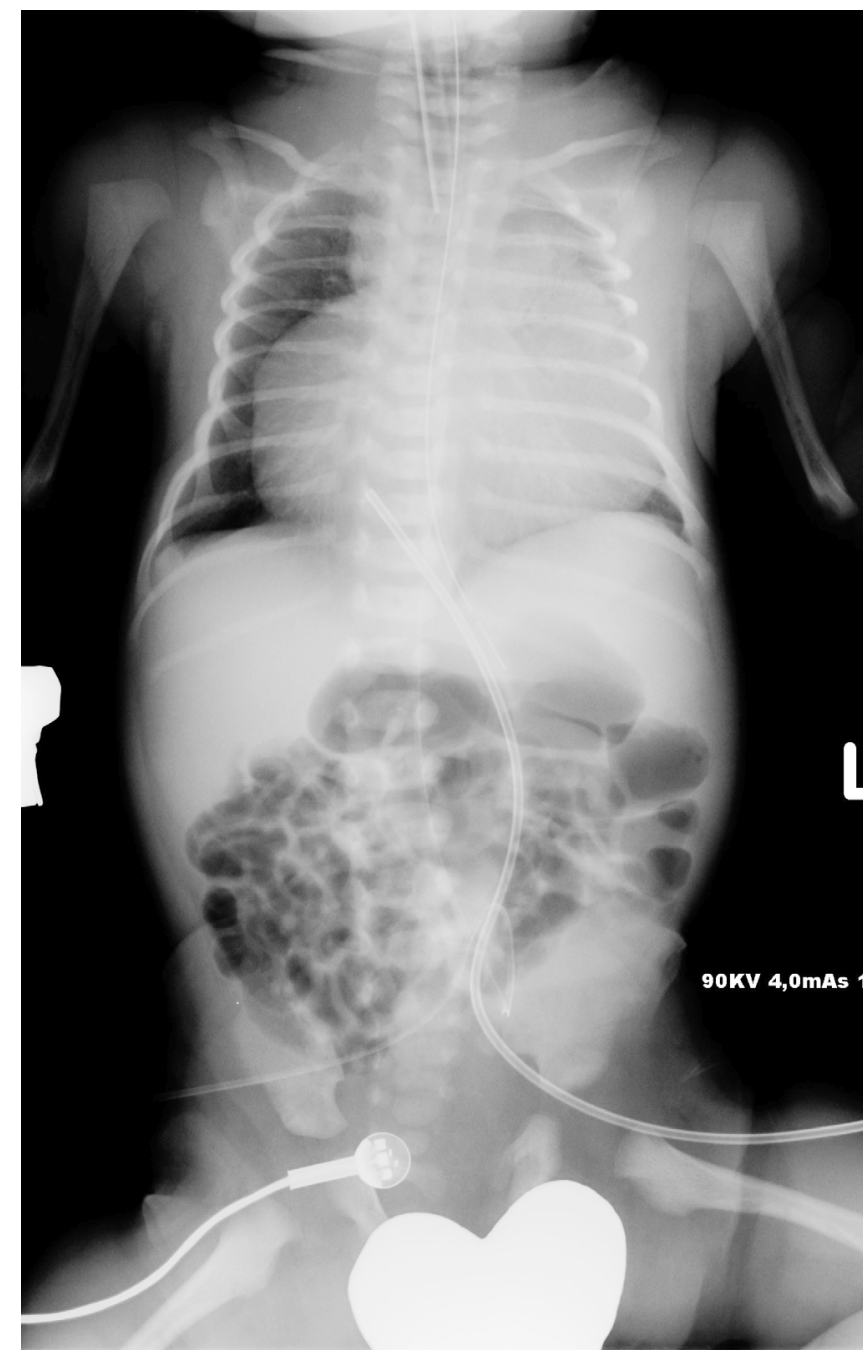

Fig. 3. X-ray image from the neonatal ICU tripled. Additionally, there were two minor markers of the possible chromosomal aberration: fetal tachycardia and hyperechogenic focus seen during the first and second trimester, respectively. All those data still showed a higher risk of amniocentesis known at that time (1\%) than the risk of severe chromosomal abnormality. The reason why the patient, who had a moderate risk of trisomy 13 , was not offered an option to have a non-invasive DNA test ${ }^{(11)}$ is unknown. She was diagnosed in the fetal cardiology center in the third trimester of pregnancy. The aim of this case report is to show the atypical appearance of right-sided diaphragmatic hernia with $\mathrm{T} 13$. In the case described, the pericardial effusion coexisted with a pericardial tumor. Such a coincidence - according to our knowledge - has never before been described in a fetus with trisomy 13 . Therefore, during fetal echocardiography and the third trimester obstetrical scan the physicians considered mainly the risk of a cardiac tamponade, not a possibility of a chromosomal abnormality ${ }^{(12)}$. The ambiguity of the overall picture was also caused by the unusual features of the pericardial tumor. The available literature had not reported a case of a prenatally diagnosed liver herniation mimicking a pericardial tumor with a pericardial effusion.

Trisomy 13 pregnancies are often associated with an older maternal age, increased fetal NT, decreased maternal serum b-hCG and PAPP-A, and fetal tachycardia ${ }^{(13-16)}$. Nonetheless, atypical presentations have also been described ${ }^{(17,18)}$.

For that reason, complete prenatal karyotyping is crucial to predict a possible outcome. It gives the mother some additional time, so that she can decide whether she wants to continue the pregnancy or not, ask for a second opinion, and receive psychological support. Doctors have appropriate knowledge to plan a delivery and postnatal treatment if the mother decides to continue with the pregnancy. If a

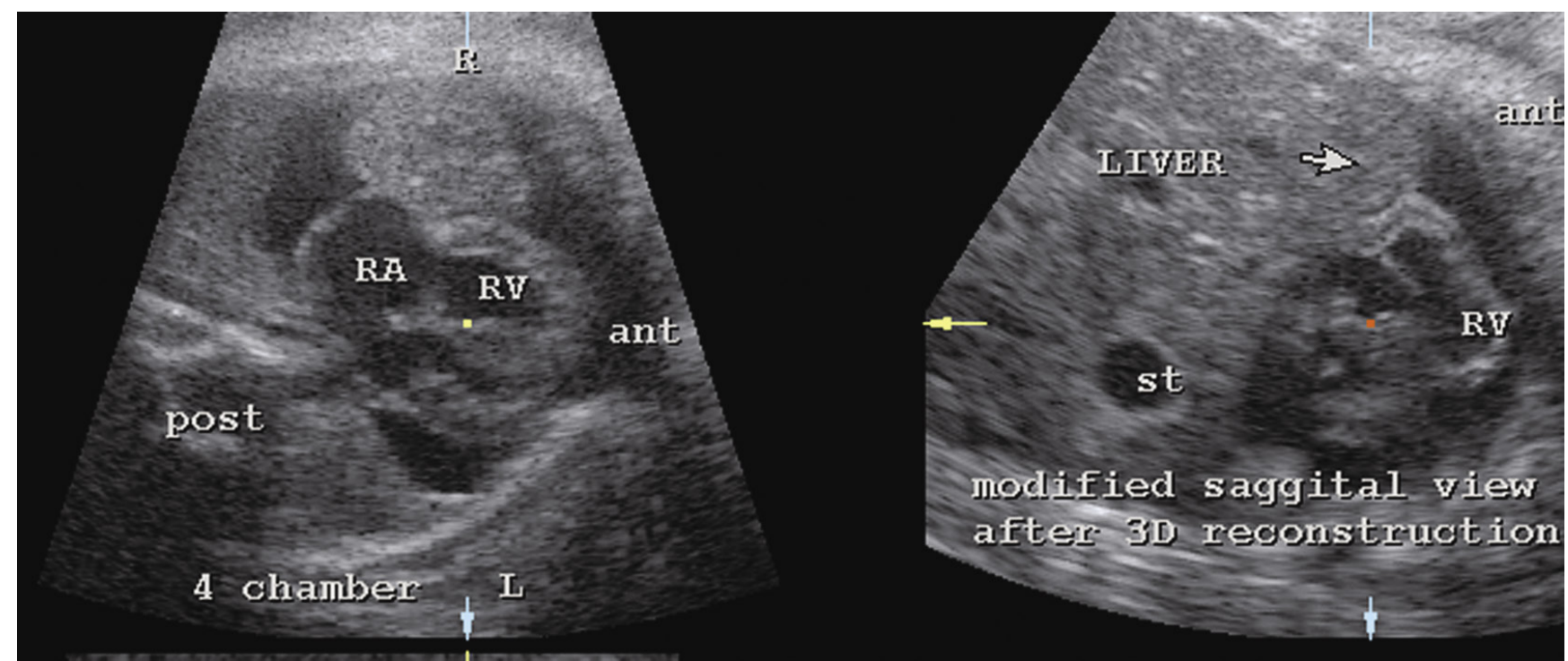

Fig. 4. Retrospective offline reconstruction from the echocardiography examination. Left-side picture: referral image in which the pericardial tumor with pericardial effusion is seen (corresponding to the Fig. 1). ant-anterior, post-posterior, RA - right atrium, RV-right ventricle, $L$-left, $R$-right. Right-side picture: modified sagittal view reconstruction from the STIC volume. The "tumor" is identified as part of the liver herniating into the pericardial cavity (arrow). st-stomach 
lethal karyotype is diagnosed, then perinatal palliative care can be offered to the parents. In the case described, if the karyotype had been known before the delivery, the mother and doctors would have had sufficient time to decide about the surgery and other invasive procedures.

\section{Conclusion}

1. Trisomy 13 can have a very atypical and benign presentation on prenatal ultrasonography, so good understanding of the first trimester screening tests, with US and biochemical results, are mandatory to provide the best care to pregnant women.

\section{References}

1. Rasmussen SA, Wong LY, Yang Q, May KM, Friedman JM: Populationbased analyses of mortality in trisomy 13 and trisomy 18. Pediatrics 2003; 111: 777-784.

2. Parker MJ, Budd JL, Draper ES, Young ID: Trisomy 13 and trisomy 18 in a defined population: epidemiological, genetic and prenatal observations. Prenat Diagn 2003; 23: 856-860.

3. Snijders RJM, Sebire NJ, Nicolaides KH: Maternal age and gestational age-specific risk for chromosome defects. Fetal Diagn Ther 1995; 10: 356-367.

4. Taslimi MM, Acosta R, Chueh J, Hudgins L, Hunter K, Chitkara U; Detection of sonographic markers of fetal aneuploidy depends on maternal and fetal characteristics. J Ultrasound Med 2005; 24 : $811-815$.

5. Lehman CD, Nyberg DA, Winter TC, Kapur RP, Resta RG, Luthy DA: Trisomy 13 syndrome: prenatal US findings in a review of 33 cases. Radiology 1995; 194: 217-222.

6. NHS Fetal Anomalous Screening Programme: Chorionic villus sampling (CVS) and amniocentesis leaflet update and published in html. FASP 2020; https://phescreening.blog.gov.uk/2020/02/28/cvs-aminocentesis-leaflet-html/.

7. Royal College of Obstetricians and Gynaecologists (RCOG): Chorionic villus sampling and amniocentesis. Information for you. RCOG Press: London, 2011. https://www.rcog.org.uk/en/patients/patient-leaflets/ amniocentesis-and-chorionic-villus-sampling/.

8. American College of Obstetricians and Gynecologists: ACOG Practice Bulletin No. 88, December 2007. Invasive prenatal testing for aneuploidy.. Obstet Gynecol 2007; 110: 1459-1467.

9. Wilson RD, Langlois S, Johnson JA: Mid-trimester amniocentesis fetal loss rate. J Obstet Gynaecol Can 2007; 29: 586-595.
2. Any atypical ultrasound image while evaluating the fetal cardiovascular system should be an indication to use more advanced methods, such as STIC, to make a proper diagnosis.

3. STIC is not just a tool to obtain a beautiful image of the fetus, but an important method to establish the final diagnosis.

\section{Conflict of interest}

Authors do not report any financial or personal connections with other persons or organizations, which might negatively affect the contents of this publication and/or claim authorship rights to this publication.

10. Salomon LJ, Sotiriadis A, Wulff CB, Odibo A, Akolekar R: Risk of miscarriage following amniocentesis or chorionic villus sampling: systematic review of literature and updated meta-analysis. Ultrasound Obstet Gynecol 2019; 54: 442-451.

11. Cell-free fetal DNA testing in prenatal genetic screening. Polish Gynaecological Society and Polish Human Genetics Society guidelines. Ginekol Pol 2015; 86: 966-969.

12. Salmanian B, Shamshirsaz AA, Cass DL, Javadian P, Ruano R, Ayres NA et al.: Fetal cardiac tamponade in a case of right-side congenital diaphragmatic hernia. Obstet Gynecol 2014; 123: 447-450.

13. Snijders RJ, Sebire NJ, Nayar R, Souka A, Nicolaides KH: Increased nuchal translucency in trisomy 13 fetuses at $10 \pm 14$ weeks of gestation. Am J Med Genet 1999; 86: 205-207.

14. Spencer K, Ong Ch, Skentou H, Liao AW, Nicolaides KH: Screening for trisomy 13 by fetal nuchal translucency and maternal serum free b-hCG and PAPP-A at 10-14 weeks of gestation. Prenat Diagn 2000; 20: 411-416.

15. Hyett JA, Noble PL, Snijders RJ, Montenegro N, Nicolaides KH: Fetal heart rate in trisomy 21 and other chromosomal abnormalities at 10-14 weeks of gestation. Ultrasound Obstet Gynecol 1996; 7: 239-244.

16. Liao AW, Snijders R, Geerets L, Spencer K, Nicolaides KH: Fetal heart rate in chromosomally abnormal fetuses. Ultrasound Obstet Gynecol 2000; 16: 610-613.

17. Turan O, Hirfanoglu IM, Beken S, Biri A, Efeturk T, Atalay Y: Prenatally detected congenital cystic adenomatoid malformation and postnatally diagnosed trisomy 13: case report and review of the literature. Turkish J Pediatr 2011; 53: 337-341.

18. Ki KD, Kim SR, Lee WI: Increased fetal nuchal translucency as a sole clue in the prenatal diagnosis of a fetus with trisomy 13. Fetal Diagn Ther 2009; 26: 54-56. 